\title{
Adaptive Fast Frequency Response for Power Electronic Connected Energy Sources
}

DOI:

10.1109/PTC.2019.8810597

\section{Document Version}

Accepted author manuscript

Link to publication record in Manchester Research Explorer

\section{Citation for published version (APA):}

Fradley, J., Preece, R., \& Barnes, M. (2019). Adaptive Fast Frequency Response for Power Electronic Connected Energy Sources. In IEEE 13th Powertech https://doi.org/10.1109/PTC.2019.8810597

\section{Published in:}

IEEE 13th Powertech

\section{Citing this paper}

Please note that where the full-text provided on Manchester Research Explorer is the Author Accepted Manuscript or Proof version this may differ from the final Published version. If citing, it is advised that you check and use the publisher's definitive version.

\section{General rights}

Copyright and moral rights for the publications made accessible in the Research Explorer are retained by the authors and/or other copyright owners and it is a condition of accessing publications that users recognise and abide by the legal requirements associated with these rights.

\section{Takedown policy}

If you believe that this document breaches copyright please refer to the University of Manchester's Takedown Procedures [http://man.ac.uk/04Y6Bo] or contact uml.scholarlycommunications@manchester.ac.uk providing relevant details, so we can investigate your claim.

\section{OPEN ACCESS}




\title{
Adaptive Fast Frequency Response for Power Electronic Connected Energy Sources
}

\author{
John Fradley, Robin Preece, Mike Barnes \\ School of Electrical and Electronic Engineering \\ The University of Manchester \\ Manchester, United Kingdom \\ John.fradley@manchester.ac.uk
}

\begin{abstract}
Future frequency control methods will be required to operate in faster timeframes than traditional forms of frequency control. Key to providing fast frequency response within low inertia systems will be power electronic (PE) interfaced energy sources. Along with improving the timeframe and quantity of response, they will also need to be scalable without causing detrimental impacts to other forms of dynamic system behavior. Simply allowing the PE-based fast frequency controls to respond quickly under small disturbances may lead to unwanted oscillatory behavior. To overcome this potential behavior, this paper proposes the use of an adaptive frequency control implementation that uses fast Fourier Transform (FFT) along with an adaptive algorithm to detect oscillatory behavior and adjust the time constant of a first order filter. The use of this adaptive frequency control is shown to significantly reduce oscillatory frequency control action for small disturbances, without reducing the timeframe of operation under large disturbances.
\end{abstract}

Index Terms-Frequency Stability, Fast Frequency Response, Rotor Angle Stability, Low Inertia Power System

\section{INTRODUCTION}

As the power system inertia reduces, the ability to contain the system frequency following a large disturbance diminishes. The system angular kinetic energy stored in the rotors of the synchronous generators (SG) - commonly referred to as the system inertia - provides the difference in energy immediately following a large disturbance. This release of energy from SG rotors arrests any sudden changes in frequency and prevents the derivative of the frequency from exceeding statutory limits that are imposed to prevent protective schemes from operating [1]. As system inertia diminishes, new methods for providing frequency response that can operate before existing primary frequency control schemes are required [2].

When a disturbance occurs in a high inertia system, the energy released from a SG is determined by the size of the disturbance, the electrical distance between the disturbance and the SG, and the inertia of the machine. The energy released is also shared among all of the other directly connected synchronously connected energy sources[3]. Due to reduced inertial energy, research is underway into new forms of fast frequency response involving the use of wind power plants (WPP) to release their inertial energy as in $[4,5]$. There are also studies using large scale battery storage for fast frequency containment as in $[6,7]$. It is expected that there will be numerous sizes of power electronic (PE) connected energy sources, the largest $\mathrm{PE}$ connections being transmission level connected HVDC interconnectors. A suitable PE candidate is the voltage source converter (VSC). VSC technology provides a greater level of control over the classic line commutated converter which makes it more desirable for use when also providing ancillary services such as frequency response $[8,9]$.

Future frequency response from fast operating devices using VSC operating in grid-following mode (as is expected during the transition to greater PE penetration) will have to respond based on a measured system value, predominantly the system frequency. Due to the structure and design of a VSC, they have the ability to respond in much shorter timeframes than the primary control of a SG as there are no mechanical or thermal processes which limit response speed. This makes them extremely beneficial for providing fast frequency response (FFR). As the number of renewable energy sources connected using VSC technology increases, so will the potential to provide increased levels of fast frequency response. A future concern will be the ability to provide fast frequency response with a sufficient amount of energy to contain the frequency following a large disturbance without having a detrimental impact on the system frequency and rotor angle stability following a small disturbance. It will be crucial to retain frequency and rotor angle stability following a small disturbance without compromising the ability to contain the frequency after a large disturbance.

To allow scalability of FFR devices and ensure that the frequency or rotor angle stability is not negatively impacted under large and small disturbances, a reduction on the speed or quantity of frequency containment action may be required. Any such adjustment would limit the speed of response of the FFR device and ultimately could reduce its impact on the 
frequency containment process. An ideal solution would be to adapt the operation of the FFR device depending on the situation.

This paper will introduce an adaptive frequency controller for a VSC frequency control loop. The overall aim of this research is to enable the VSC to provide FFR without causing detrimental stability impacts, particularly with consideration of small disturbances.

\section{THE NECESSITY FoR FAST FREQUENCY RESPONSE}

A common equation for stability analysis is the swing equation given by (1), where $H$ is the inertia constant, $P_{m}$ and $P_{e}$ are the mechanical and electrical power and $K_{d} \omega_{r}$ is the rotor damping component. Viewing the swing equation in (1) highlights that as the overall system inertia $(H)$ reduces, and the increase in mechanical power remains constrained by the SG physical properties, the speed of the frequency deviation known as the rate of change of frequency (ROCOF) will increase. The inevitable reduction in inertia will lead to the requirement for frequency control services to respond faster than they traditionally have done to alleviate the imbalance between mechanical and electrical power. Proportional based strategies known as droop control are incorporated into SGs with typical droop slope values in the range of $3-5 \%$. The slope of the droop in a SG is limited by the physical properties of the machine which prevents it from simply being increased to cope with faster changes in system frequency.

As future power electronic connected energy sources will not provide any natural inertial response, they will need to provide frequency response faster than the primary control of a SG. If they are to provide frequency response services using a droop based control strategy, the droop slope may need to be significantly steeper to cover some of the energy that has typically been provided by the SG kinetic energy as highlighted by [10]. FFR based on measured signals will never be able to naturally respond like the inertial response from a $\mathrm{SG}$, but with increased droop, they can provide a sufficient amount of energy to limit the ROCOF. As well as providing sufficient amounts of energy following a disturbance, the timeframe for the response to operate will also be crucial. Current expectations from the UK system operator are that FFR will start to respond within $1 \mathrm{~s}$ [2].

$$
\frac{d \omega}{d t}=\frac{1}{2 H}\left(P_{m}-P_{e}-K_{d} \omega_{r}\right)
$$

\section{VSC-HVDC FREQUENCY CONTROL}

The full cascaded VSC-HVDC control structure and its operation is reviewed in $[11,12]$. Within the cascaded control structure there are current limits and set point ramping limits, which prevent the converter from operating outside design limits. In order to enable the converter to provide frequency response, a supplementary control loop is required. The supplementary frequency control loop output provides an additional power reference $(\Delta P)$ into the outer control loop displayed in Fig.1a [13]. Limiting factors affecting the response are the phase lock loop (PLL) which measures the system frequency, the gain component $\left(K_{p}\right)$, and potentially any other delays introduced from the signal processing.

To improve the frequency response capability, the PLL bandwidth could be increased, and/or the value of $K_{p}$. For large disturbances, increasing these parameters would be beneficial as a larger quantity of energy would be injected over shorter timeframes. However, for smaller disturbances, increasing these parameters can lead to unwanted oscillatory behavior or limit cycling from the frequency control loop. Unwanted behavior occurring from the converter control loop is dependent on the value of $K_{p}$, the system inertia, and the quantity of FFR operating in the system. To highlight this behavior, four scenarios described in Table I are used. These scenarios are representative of disturbances of different size in a transmission system approximately scaled to the Great British power system at low inertia levels. These disturbances are all less than the maximum infeed loss scenarios for the GB system which is $1800 \mathrm{MW}$. The resultant outputs of the VSC frequency control loop for these scenarios are displayed in Fig.2. This highlights the potential detrimental impact of providing FFR under small disturbances. The frequency control in scenario 1 operates first because the frequency exits the set deadband faster than the other scenarios, due to it being subject to the largest disturbance.

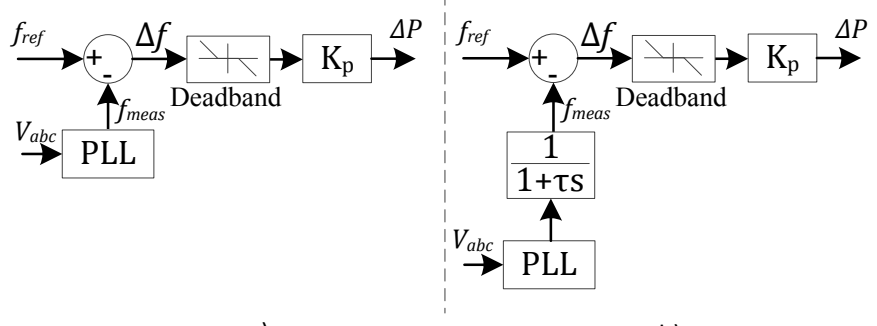

a)

b)

Fig.1. VSC-HVDC supplementary control loops

TABLE I. EXAMPLE SCENARIO PARAMETERS

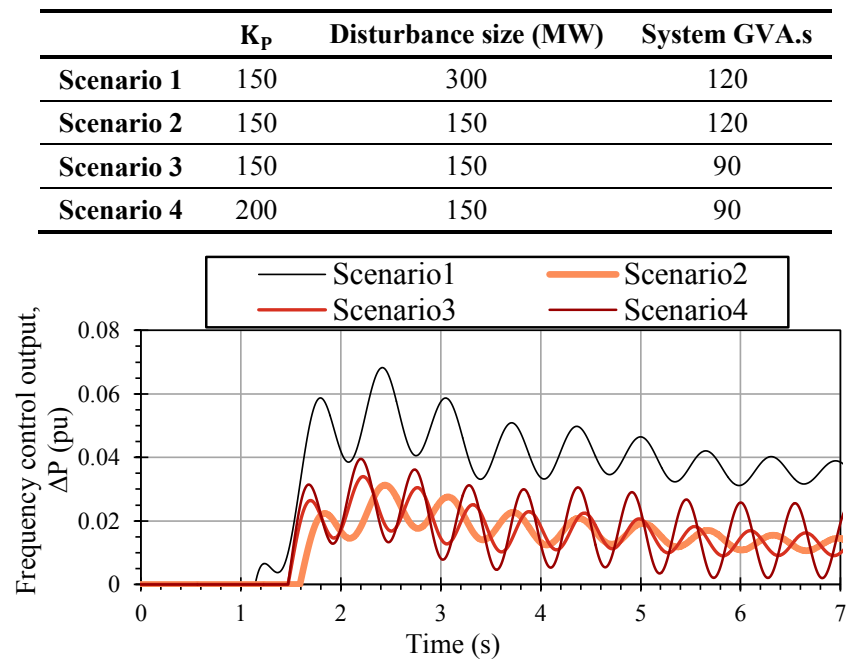

Fig.2. VSC frequency control output behavior 
To prevent this unwanted action from the converter due to the frequency control loop, the gain could be reduced or the PLL bandwidth could be reduced. These both reduce the response from the converter leading to a lower impact from the FFR. An alternative could be to introduce a first order lag filter displayed in Fig. $1 \mathrm{~b}$ for the measured frequency value to act as a band pass and limit the converter response to frequencies above a certain range. However, the introduction of a first order filter would reduce the FFR capability of the converter. Although this will have a positive impact for small disturbances in low inertia networks when multiple devices are providing FFR, it would be unfavorable for large disturbances or when there is limited FFR connected. The impact of the addition of a first order filter with a time constant of $0.5 \mathrm{~s}$ on the converter output for an example situation with a large disturbance is displayed in Fig. 3 and Fig.4.

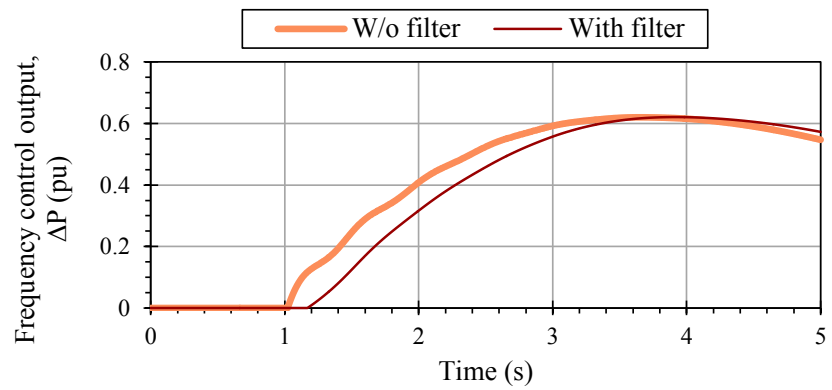

Fig.3. Impact of first order filter on converter response

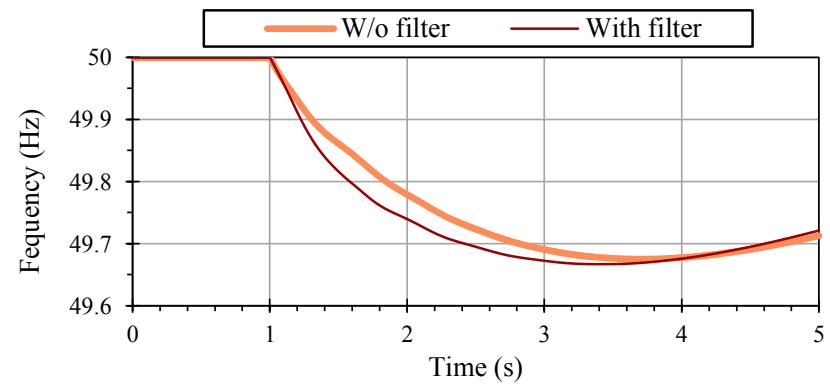

Fig.4 Impact of the first order filter on system frequency

The ability to provide FFR that is capable of being applied for large and small disturbances may become a challenge as the network changes and the number of FFR devices connected increases. Future practical implementations of FFR will need to be scalable without the need to be constantly retuned and without causing detrimental system impacts.

\section{AUGMENTED VSC-HVDC FREQUENCY CONTROLLER}

An adaptive filter has been designed in this work in order to prevent unwanted oscillatory frequency control behavior without slowing down the speed of the converter response. This adaptive filter allows the converter to provide large quantities of response for large disturbances. At the same time, if oscillatory or limit cycle behavior is detected, it varies the filter time constant to reduce or remove the undesirable behavior. The method proposed in this paper is displayed in Fig.5 and is a corrective action instead of a preventative one. The adaptive controller detects any oscillating or limit cycle behavior in the output of the frequency control loop and adjusts the time constant of the first order filter. The dominant frequency detected is used to determine a time constant for the first order filter. To prevent any sudden filter operation changes, the new time constant is ramped up to the new value with a gradient limit of $1 \mathrm{~s} / \mathrm{s}$. Once any output oscillations have settled down, the filter time constant is returned to its original value enabling the controller to respond as fast as possible once again.

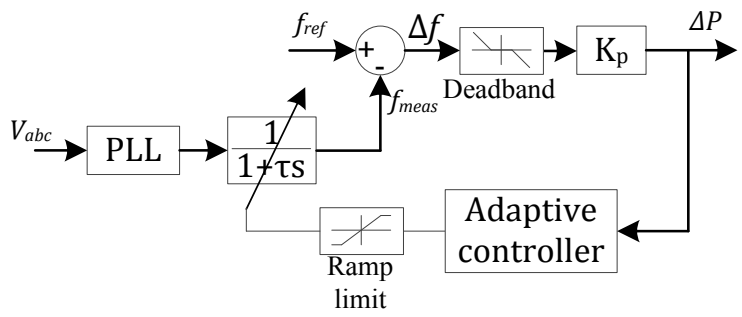

Fig.5. Proposed FFR control method

Detecting any oscillations in the output of the frequency controller could be achieved using a number of different methods. In this research, the detection is completed using a discrete Fourier transform given by (2) where $k$ is an individual sample, $N$ is the number of samples and $T$ is the time sample separation. To improve computing time, the discrete Fourier transform is achieved by applying a fast Fourier transform (FFT) algorithm. The chosen sampling rate is $500 \mathrm{~Hz}$ and the number of samples is 2000, producing a resolution of $0.25 \mathrm{~Hz}$. The output vector from the FFT that contains the transformed time domain signal information is passed to the adaptive algorithm displayed in Fig.6. The high frequency components from the measured frequency signal are expected to have been filtered out due to the PLL bandwidth, which does not have to be as large for the frequency measurement as for the converter synchronization. The adaptive control algorithm determines the frequency with the largest magnitude that is detected by the FFT and based on that, sets a time constant for the first order filter. The FFT output only occurs when a set number of samples have been processed which requires a compromise between the speed that the adaptive control can adjust for any oscillations due to the controller and the precision of the detected frequencies in the output. Taking this into consideration, once the adaptive algorithm has determined the frequency with the largest magnitude, it calculates the time period of that frequency $\left(T_{\max }\right)$ and determines the filter time constant as (3) which is used to overcompensate for any errors at the detection stage. The value of $\tau_{s}$ is expected to be in the range of $0-2 \mathrm{~s}$ but is dependent on the system dynamics. More elaborate methods could be applied at this point to improve the decision making and tuning of the value for $\tau s$, but at this point a simple approach has been applied.

$$
\mathrm{F}=\sum_{k=0}^{N-1} f[k] e^{-j w k T}
$$




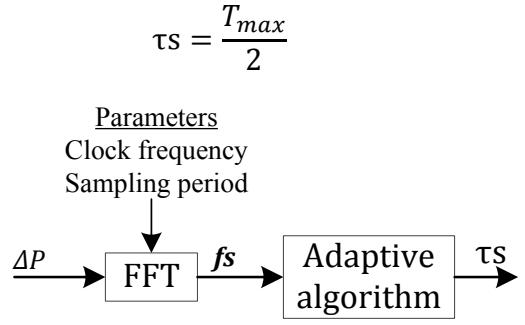

Fig.6. Adaptive controller

\section{TEST SySTEM}

In order to investigate the adaptive FFR scheme, a generic four area test system has been created as displayed in Fig.7. Each area has an aggregated SG, WPP and load. Area 1 has an aggregated VSC that encompasses the VSC frequency control loop. The test system has been created to be representative of a future low inertia system.

In this test system scenario, synchronous generation makes up $40 \%$ and non-synchronous generation makes up $60 \%$ of the load with the parameters for each area given in Table II. Each SG operates with an IEEET1 AVR and an IEEEG1 governor. All transmission lines have an impedance of $0.375+\mathrm{j} 3.75 \Omega$. The average value VSC model incorporates dq-vector control which has inner and outer loops. The disturbance to create the frequency stability event is the disconnection of a proportion of non-SG in area 4. Different test cases will explore different sizes of disconnection. The total system inertia is 120 GVA.s with each SG having an inertia constant $(H)$ of $4 \mathrm{~s}$. The SG governor models do not incorporate a dead band, but the dead band is implemented in the VSC frequency control loop and is $+/-15 \mathrm{mHz}$. The available FFR from the VSC is 1000 MW. This is representative of an aggregation of multiple FFR services which could not only include interconnectors, but also large scale battery storage.

The test system has been implemented in DIgSILENT Powerfactory 2017 and the adaptive algorithm has been implemented in MATLAB R2016a. DIgSILENT interfaces to MATLAB every simulation time step in order to achieve the relevant calculations. The simulation time step for the RMS simulation is $1 \mathrm{~ms}$ which allows a clock frequency of $500 \mathrm{~Hz}$ which is used for the FFT sampling signal.

TABLE II. TOTAL DisPatched GENERATION AND LOAD

\begin{tabular}{lccccc}
\hline & Area1 & Area2 & Area3 & Area4 & Total \\
\hline SG (GW) & 5 & 5 & 5 & 5 & 20 \\
WPP (GW) & 3.5 & 7.5 & 7.5 & 7.5 & 26 \\
VSC (GW) & 4 & - & - & - & 4 \\
Load (GW) & 12.5 & 12.5 & 12.5 & 12.5 & 50 \\
\hline
\end{tabular}

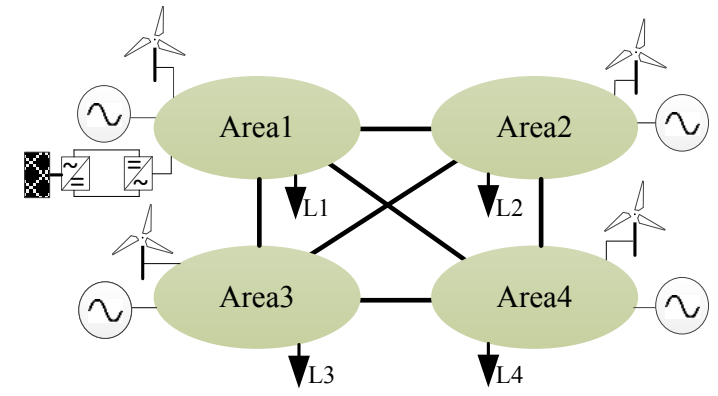

Fig.7. Generic four-area test system

\section{RESULTS}

The results of the adaptive control are compared to cases without the adaptive control to ascertain if there are any benefits. There are an extensive number of test cases that could be applied including changing the level of system inertia, applying different levels of FFR, and varying FFR response times. However, at this stage in the research, four different cases have been selected. Test cases 1 and 2 investigate the impact of the controller action following small disturbances. Test case 3 investigates the controller action following a large disturbance to view if it impacts the converters ability to provide FFR. Test case 4 investigates the impact of medium scale disturbance.

\section{A. Test case 1}

The first test case for comparison has a disturbance size of $150 \mathrm{MW}$ and a VSC frequency control gain $\left(k_{p}\right)$ of 150 with a view of investigating how the controller can reduce oscillatory output behavior due to the FFR. The VSC frequency control loop output $(\Delta P)$ is displayed in Fig.8 and compares the output without the adaptive control and with the adaptive control. When the frequency control loop activates there is no difference in the speed of the response and both control types lead to an initial output oscillation. However, the adaptive control detects the oscillation and applies a time constant to the first order filter which reduces the frequency pass band of the filter. As the disturbance is relatively small, the overall frequency deviation is minimal. The adaptive control prevents sustained injection of power oscillations being injected into the network and causing frequency oscillations as displayed in Fig.9. Depending on network topology and rotor angle stability factors, these oscillations could lead to rotor angle stability issues.

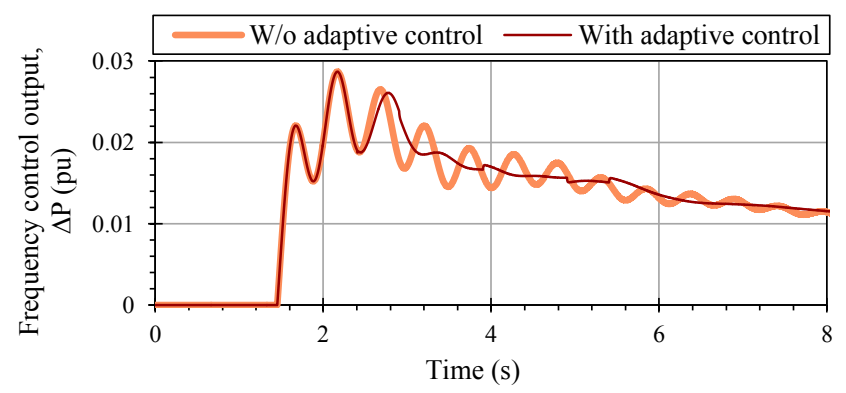

Fig.8. VSC frequency control output for test case 1 


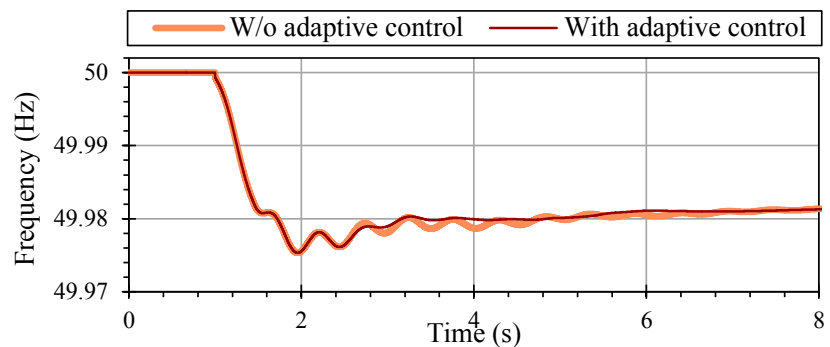

Fig.9. System frequency for test case 1

The first order filter time constant value is displayed in Fig. 10 and shows the value is increased via a ramp when the adaptive controller detects oscillatory behavior. The filter value is reduced back to zero by the adaptive controller as the oscillations decrease. In this study the adaptive controller and FFT setup does not have the resolution to distinguish between the subtle frequency changes between each case, this leads to a similar filter time constant profile for each case.

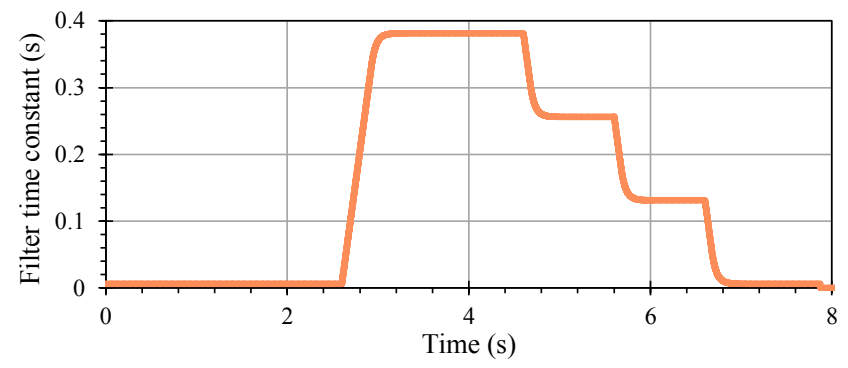

Fig.10. Filter time constant for test case 1

\section{B. Test case 2}

For the second test case, the frequency control gain is increased to 175 but all other parameters are the same as test case 1 . In this test case, due to the larger value of gain applied, the control action is larger leading to greater oscillation magnitude without adaptive control as displayed in Fig.11. Similar to test case 1, both the non-adaptive and adaptive control initially respond in the same manner to begin until the adaptive control has detected the oscillations in the controller output. In this test case, without the adaptive frequency control, larger power oscillations would be injected into the network as displayed in Fig. 12 which would increase the risk of rotor angle stability issues. In this particular case, without the adaptive controller the oscillations were not reducing or being damped out.

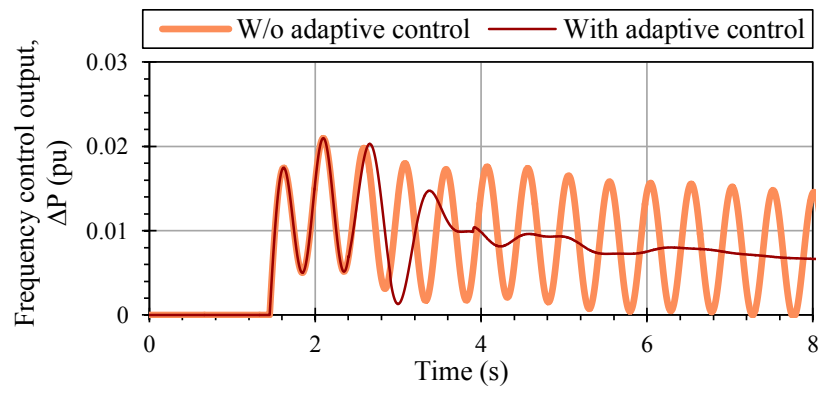

Fig.11. VSC frequency control output for test case 2

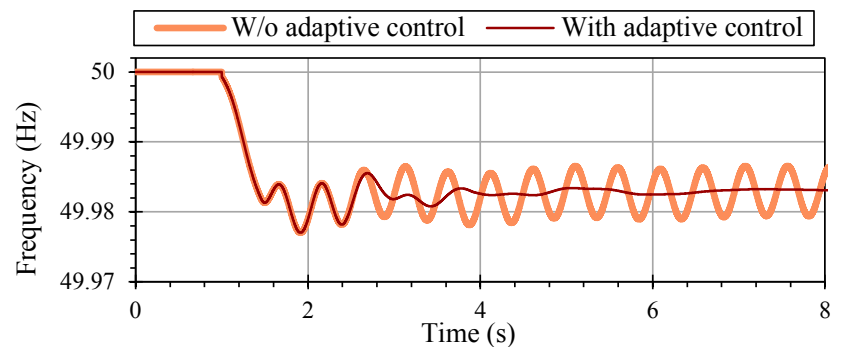

Fig.12. System frequency for test case 2

\section{Test case 3}

This third test case investigates the influence of the adaptive control when a large disturbance occurs. The aim of the adaptive control is to not reduce the speed of the response when a large disturbance occurs. This test case will examine if the adaptive control reduces the speed of response which could lead to an increased rate of change of frequency or worse frequency nadir. In this test case the disturbance size is $1800 \mathrm{MW}$ and the VSC frequency control gain value is 150 .

In Fig.13, the non-adaptive and the adaptive VSC frequency control outputs do vary slightly. The FFT has detected some small oscillatory action and has adapted the first order filter time constant. It is important to remember that although the frequency control loop may be large, the converter output is limited by the inner and outer control loops depending on head room. This detection of a slight oscillation and the subsequent change in the filter time constant does not reduce the ability of the converter to provide FFR for large disturbances as displayed in Fig.14 where no difference in ROCOF or frequency nadir is seen. The impact of FFR with and without adaptive control is also compared against a scenario where no FFR is applied in order to show that the FFR does have a system impact.

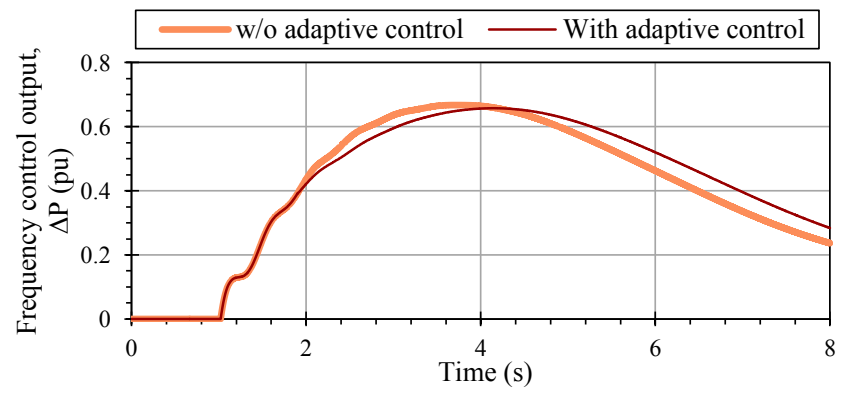

Fig.13. VSC frequency control output for test case 3

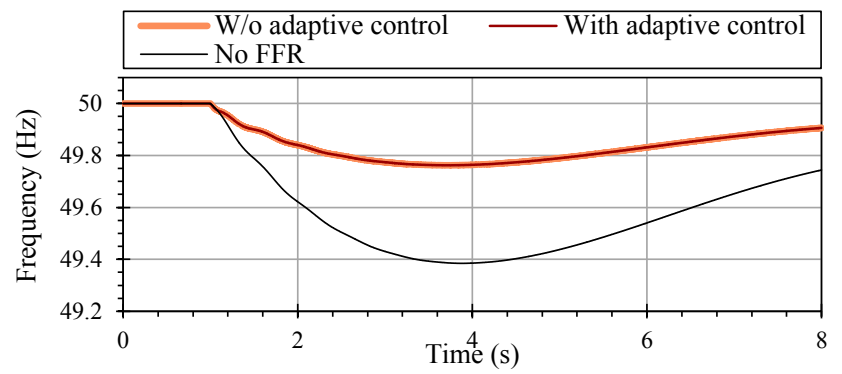

Fig.14. System frequency for test case 3 


\section{Test case 4}

The fourth test case is presented to highlight what impact the adaptive controller has when a medium size disturbance occurs on the network. In this test case the disturbance size is $500 \mathrm{MW}$ allowing the frequency deviation to be more significant than case 1 and 2, but not as severe as case 3 .

It is observed in Fig.15 that similar to test cases 1 and 2, due to the high gain value of the FFR, oscillations are introduced. In Fig. 16 the adaptive controller is shown to allow initial frequency containment the same as without the adaptive controller. This displays the adaptive controller does not reduce the speed or quantity of initial power injection. Following the initial containment, the adaptive frequency controller is shown to damp out any unwanted oscillations introduced by the FFR in Fig. 16.

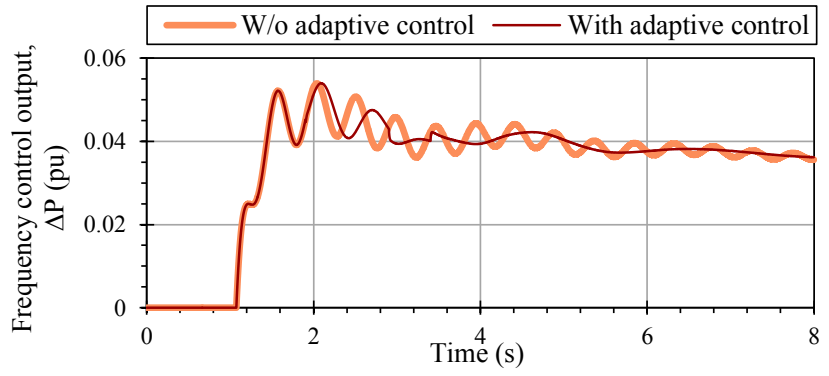

Fig.15. VSC frequency control output for test case 4

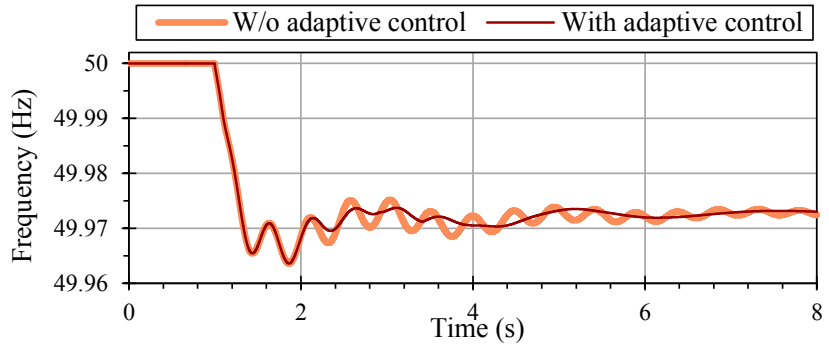

Fig.16. System frequency for test case 4

\section{CONCLUSION}

This research paper has re-emphasized the need for FFR schemes for future low inertia power systems. It has also highlighted that certain factors, namely, system inertia, FFR response time, FFR power injection quantity, and disturbance size, could create situations where the FFR schemes inject unwanted power oscillations into the network. This is expected to be more prevalent when there is an increasing quantity of FFR connected to the network and the network is subject to small disturbances.

To enable larger quantities of FFR to be connected to future low inertia systems, without leading to the situation where unwanted oscillatory action is introduced; this paper has presented a simple adaptive FFR control scheme for a VSC system. The adaptive control process utilizes a FFT method to detect oscillations in the output of the frequency controller. A resultant time constant determined by an adaptive algorithm is then applied to a first order filter on the frequency measurement signal in order to reduce the injection of oscillations by the frequency controller. This simple adaptive control method has been shown via simulation to enable the converter to respond quickly (the same as with no adaptive control) to large disturbances. Under small disturbances, the adaptive controller reduces the unwanted power oscillations introduced.

The test cases presented have highlighted how an adaptive frequency control scheme could be implemented to allow a greater quantity of FFR to be connected to the network by limiting the impact following low disturbance scenarios. Future work will investigate more efficient algorithms to detect unwanted frequency control behavior as the FFT is limited by the sample frequency and number of samples. Future work will also involve more robust methods for assigning the filter time constant based on the detected oscillatory behavior.

\section{ACKNOWLEDGMENT}

The authors would like to thank Siemens and the EPSRC for providing guidance and financial support during this work through the EPSRC Centre for Doctoral Training in Power Networks (EP/L016141/1)

\section{REFERENCES}

[1] National Grid UK, "System Operability Framework 2016," Tech. Rep., Nov. 2016.

[2] National Grid UK, "Product Roadmap: For frequency response and reserve," Tech. Rep., Dec. 2017.

[3] J. Machowski, J. Bialek, J.Bumby, Power system Dynamics: Stability and Control: John Wiley and Sons, 2008.

[4] H. T. Nguyen, G. Yang, A. H. Nielsen, and P. H. Jensen, "Combination of Synchronous Condenser and Synthetic Inertia for Frequency Stability Enhancement in Low Inertia Systems," IEEE Transactions on Sustainable Energy, in press (early access), 2018.

[5] F. Gonzalez-Longatt, E. Chikuni, and E. Rashayi, "Effects of the Synthetic Inertia from wind power on the total system inertia after a frequency disturbance," in Proc. IEEE Ind. Tech. International Conf., Cape Town, South Africa, Feb. 2013.

[6] Y. Kim, "Experimental study of battery energy storage systems participating in grid frequency regulation," in Proc IEEE Transmission and Distribution Conference and Exposition, Dallas, USA, May 2016.

[7] D. M. Greenwood, K. Y. Lim, C. Patsios, P. F. Lyons, Y. S. Lim, and P. C. Taylor, "Frequency response services designed for energy storage," Applied Energy, vol. 203, pp. 115-127, Oct. 2017.

[8] A. S. Elansari, S. J. Finney, J. Burr, M. F. Edrah, "Frequency control capability of VSC-HVDC transmission system," in Proc. 11th IET International Conf. on $A C$ and $D C$ Power Transmission, Beijing, China, July 2015.

[9] National Grid UK, "Benefits of Interconnectors to GB Transmission System," Tech. Rep., Dec. 2014.

[10] EIRGRID, SONI, "RoCoF Alternative \& Complementary Solutions Project,". [Online]. Available: http://www.eirgridgroup.com/sitefiles/library/EirGrid/RoCoF-Alternative-Solutions-Project-Phase-2Report-Final.pdf, [Accessed 26 ${ }^{\text {th }}$ Sep. 2018], 2016.

[11] R. Shah, J. C. Sánchez, R. Preece, and M. Barnes, "Stability and control of mixed AC-DC systems with VSC-HVDC: a review," IET Generation, Transmission \& Distribution, vol. 12, May 2018.

[12] K. Sharifabadi, L. Harnefors, H.-P. Nee, S. Norrga, and R. Teodorescu, Design, Control and Application of Modular Multilevel Converters for HVDC Transmission Systems, John Wiley \& Sons, 2016.

[13] C. E. Spallarossa, Y. Pipelzadeh, and T. C. Green, "Influence of frequency-droop supplementary control on disturbance propagation through VSC HVDC links," in Proc. IEEE Power \& Energy Society General Meeting, Vancouver, Canada, July 2013. 